\title{
Fast Gated Imaging of the Collisionless Interaction of a Laser-Produced and Magnetized Ambient Plasma
}

\author{
P. V. Heuer ${ }^{\mathrm{a}}$, D. B. Schaffer ${ }^{\mathrm{a}}$, E. N. Knall ${ }^{\mathrm{a}}$, C. G. Constantin ${ }^{\mathrm{a}}$, L. R. Hofer ${ }^{\mathrm{a}}$, S. Vincena ${ }^{\mathrm{a}}$, S. Tripathi ${ }^{\mathrm{a}}$, C. \\ Niemann ${ }^{\mathrm{a}}$ \\ ${ }^{a}$ University of California Los Angeles
}

\begin{abstract}
The collisionless interaction between a laser-produced carbon plasma (LPP) and an ambient hydrogen plasma in a background magnetic field was studied in a high shot rate experiment which allowed large planar data sets to be collected. Plasma fluorescence was imaged with a fast-gated camera with and without carbon line filters. The resulting images were compared to high-resolution two dimensional (2D) data planes of measured magnetic field and electric potential. Several features in the fluorescence images coincide with features in the field data. Relative intensity was used to determine the initial angular velocity distribution of the LPP and the growth rate of instabilities. These observations may be applied to understand fluorescence images from similar experiments where 2D planes of field data are not available.
\end{abstract}

\section{Introduction}

Magnetized collisionless shocks form in many space and astrophysical environments where one plasma encounters another plasma (supernovae remnants [1], coronal mass ejections [2]) or an obstacle (bow shocks) at a speed faster than the local wave propagation speed (here the Alfvén speed). The shock forms as particles in the plasma interact only through the bulk electromagnetic fields, forming a complex and non-trivially three dimensional macroscopic shock structure.

Collisionless magnetized shocks are often studied in the laboratory by observing the interaction between a laser-produced plasma (LPP) and an ambient background plasma $[3,4]$. As the LPP expands, currents along the edge expel the background magnetic field, creating a diamagnetic cavity or "bubble" that serves as an electromagnetic piston to drive a shock in the ambient plasma $[5,6]$. High laser energies are required to produce the large pistons necessary to drive super Alfvénic shocks, limiting experiments to relatively small data sets. High shot rate experiments at lower energies complement these experiments by collecting large data sets to Heuer)

Email address: pheuer@physics.ucla.edu (P. V.

Preprint submitted to Elsevier validate simulations which can then be scaled to high energy.

Fast-gated fluorescence imaging is a non-invasive diagnostic technique that captures a 2D image of plasma self-emission over a short $(<10 \mathrm{~ns})$ exposure period $[7,8]$. Since self-emission intensity is dependent on the plasma density, these images provide some information about the density distribution. Imaging over successive shots allows the $2 \mathrm{D}$ velocity distribution of the emitting plasma to be calculated by time of flight.

A recently commissioned high-frequency laser at the University of California, Los Angeles (UCLA) was paired with the ambient plasma of the Large Plasma Device (LAPD) to collect both fast gate fluorescence images and 2D planes of vector field data at repetition rates of up to $1 \mathrm{~Hz}$. The resulting data sets match previously performed hybrid simulations [9] and suggest correlations between fluorescence features and field features that may be useful in diagnosing future higher energy experiments.

\section{Experimental Setup}

Experiments were carried out with a highrepetition laser on the Large Plasma Device (LAPD) at UCLA. The laser $(1053 \mathrm{~nm}, 20 \mathrm{~J}, 15$ 


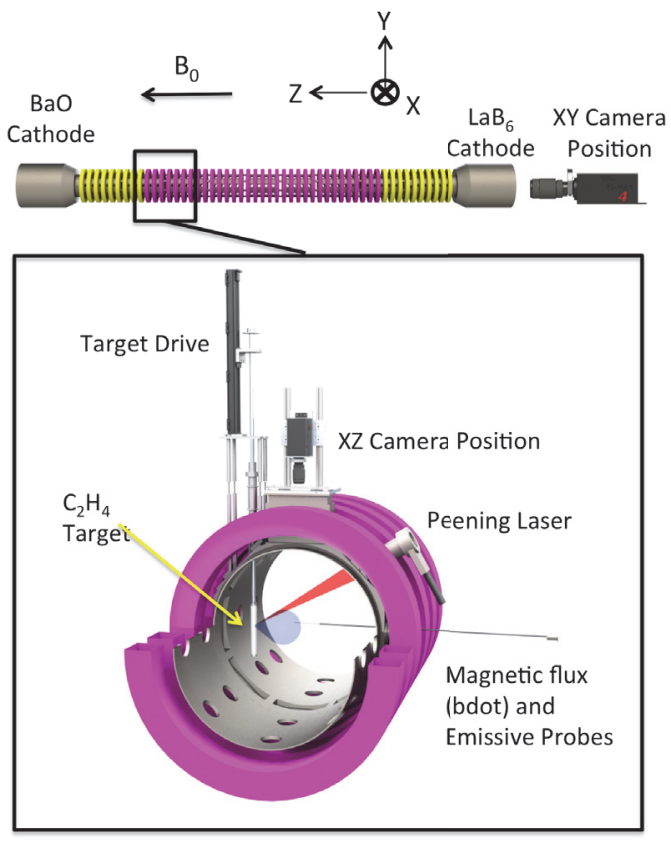

Figure 1: Experimental setup on the LAPD.

ns FWHM), is part of the Phoenix Laser Laboratory at UCLA. The laser was fired at a repetition rate of $0.25 \mathrm{~Hz}$ and focused to an intensity of $10^{13}$ $\mathrm{W} / \mathrm{cm}^{2}$ onto a cylindrical high density polyethylene ( $\mathrm{HDPE}, \mathrm{C}_{2} \mathrm{H}_{4}$ ) target embedded in an ambient plasma. The laser entered the chamber through a focusing lens at $30^{\circ}$ to the target normal. The LPP expands perpendicular to the target surface regardless of the angle of incidence. The target was rotated and translated between shots to provide a fresh surface.

The ambient plasma was created in the LAPD [10], operated by the Basic Plasma Science Facility (BaPSF). The LAPD is a $20 \mathrm{~m}$ long and $1 \mathrm{~m}$ diameter solenoidal plasma device. An axial background magnetic field of $250 \mathrm{G}$ is created by a series of electromagnets (purple rings in Fig. 1). An ambient hydrogen plasma with a partial pressure of $2.9 \times 10^{-5}$ Torr is created by cathodes at opposite ends of the chamber: a $\mathrm{BaO}$ (barium oxide) coated cathode creates a low-density plasma $\left(n \approx 2 \times 10^{12}\right.$ $\mathrm{cm}^{-3}$ ) $60 \mathrm{~cm}$ in diameter, while a $\mathrm{LaB}_{6}$ (lanthanum hexaboride) cathode creates a smaller, higher density plasma $\left(n \approx 2 \times 10^{13} \mathrm{~cm}^{-3}\right) 40 \mathrm{~cm}$ in diameter. The resulting plasma is highly repeatable, steady state (for $10 \mathrm{~ms}$ ), quiescent, and current free. The LAPD was operated at $0.25 \mathrm{~Hz}$ and synchronized with the high-repetition laser.

\begin{tabular}{lcc} 
Ion & Line $\lambda(\mathrm{nm})$ & Filter Center $\lambda(\mathrm{nm})$ \\
\hline CIII $\left(C^{+2}\right)$ & 418.7 & 410 \\
CIV $\left(C^{+3}\right)$ & 444.2 & 450 \\
CV $\left(C^{+4}\right)$ & 494.8 & 500
\end{tabular}

Table 1: Filters used for each ion species.

The axially directed background magnetic field defines the $\hat{z}$ axis, while the target normal defines the $\hat{x}$ axis. The axis tangent to the target surface defines $\hat{y}$ (Fig. 1). The origin is defined as the point where the laser hits the target. The laser shot on target defines $\mathrm{t}=0$.

Fast gated images were taken using a Princeton Instruments PI-MAX 4 intensified CCD (ICCD) camera. The camera exposure was set to $10 \mathrm{~ns}$. In order to image successive time steps, the camera trigger was delayed by $0-2 \mu$ s after the laser pulse in steps of 10 ns. Each time step was repeated over multiple laser shots for reproducibility and noise averaging. For some shots, FWHM $10 \mathrm{~nm}$ bandpass filters were used to isolate desired emission lines (see Table 1).

Images were taken through Pyrex ${ }^{\circledR}$ glass windows (transparent at measured wavelengths) from two camera positions. For images of the xz plane, the camera was mounted with a wide-angle lens ( $f=8 \mathrm{~mm}$ ) above the LAPD looking down the $\hat{y}$ axis (perpendicular to the background magnetic field), centered at $\mathrm{x}=0$ (see Fig. 1). No distortions were observed due to the lens in the region of interest. Images of the xy plane were taken using a zoom lens $(f=70 \mathrm{~mm}-300 \mathrm{~mm})$ from the far end of the LAPD. The camera was aligned along the $+\hat{z}$ axis (parallel to the background magnetic field) and centered at $\{x, y\}=\{0,0\}$.

Magnetic field measurements were made using a 3 axis, differentially wound magnetic flux "B-dot" probe, $1 \mathrm{~mm}$ in diameter [11]. Integrating the induced current in the three sets of coils, together with the known background field, provided the vector magnetic field as a function of time. 2D data planes were assembled by moving the probe in a grid pattern over successive laser shots. The resulting data was then used to compute the vector electrostatic current density.

Electric potential measurements were made using an emissive probe [12]. An exposed element within the plasma was heated to thermionic emission, at which point it equilibrated with the plasma potential, allowing the latter to be measured. 2D 


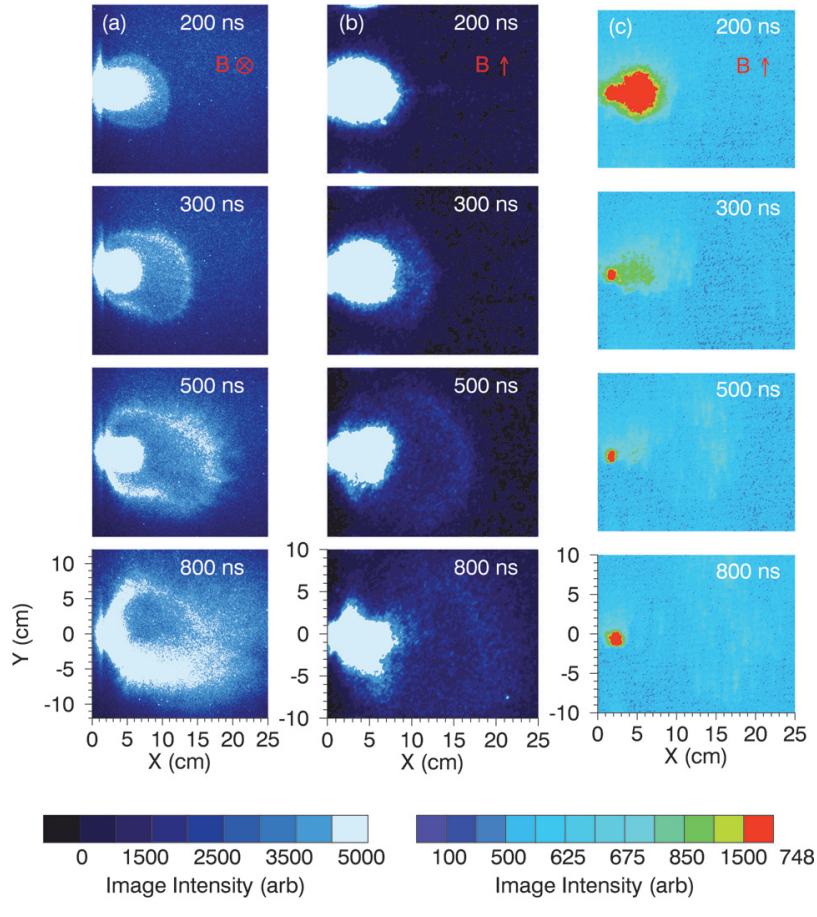

Figure 2: (a) Unfiltered fluorescence images in the xy plane show formation and growth of shell. Finger ('flute') instabilities are visible at $500 \mathrm{~ns}$. (b) Unfiltered fluorescence images in the $\mathrm{xz}$ plane. (c) Filtered fluorescence images at $500 \mathrm{~nm}$ $(\mathrm{CV})$ in the $\mathrm{xz}$ plane. Fluorescence in the shell is visible in the last two timesteps.

spatial planes were again created by moving the probe between shots. The gradient was then taken to compute the vector electric field.

\section{Fast-Gated Fluorescence Images}

Light emitted from the plasma at an atomic transition wavelength was assumed to be either fluorescence or continuum radiation such as bremsstrahlung or radiative recombination. In spectroscopic measurements in a previous experiment, no continuum emission was observed at distances far from the target. This implies that the dominant source of emitted visible light away from the target in these experiments was ion fluorescence. The intensity of a transition line from quantum numbers $\mathrm{n}$ to $\mathrm{m}$ is [13]:

$$
I_{m n}=\frac{A(n, m) h \nu_{0} N_{n}}{4 \pi} d
$$

Where $A(n, m)$ is the probability of a $n \rightarrow m$ transition, $\nu_{0}$ is the frequency of the associated spectral
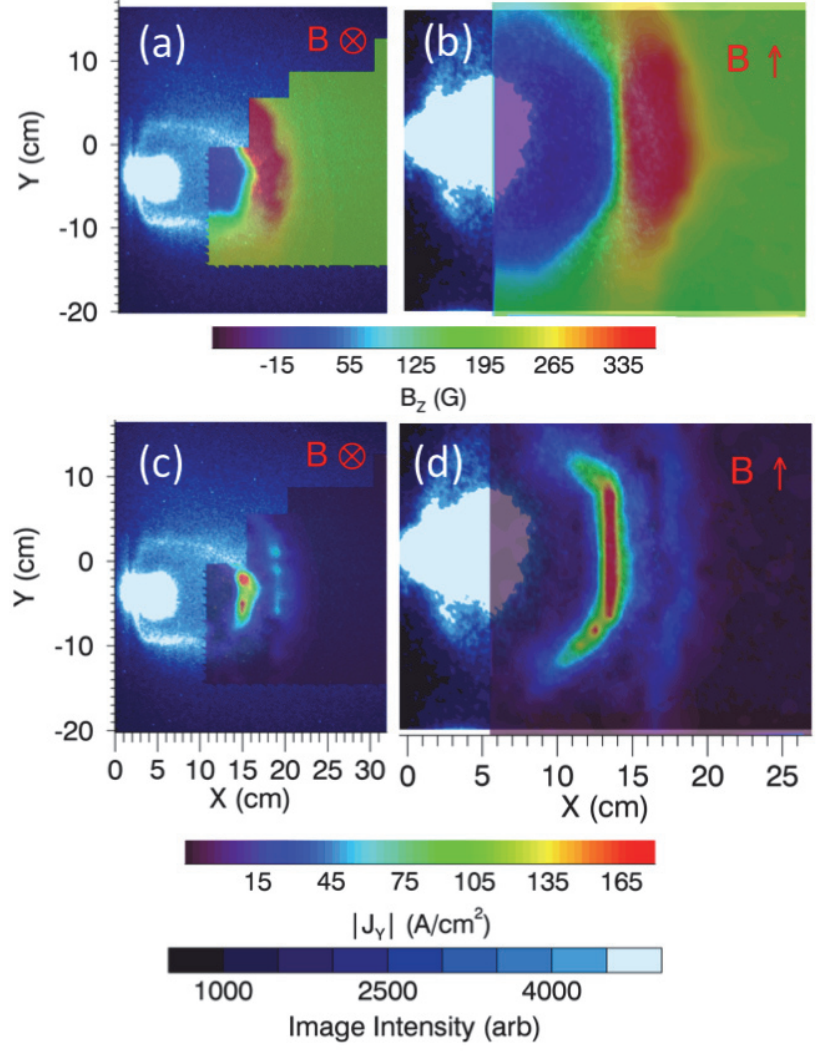

Figure 3: (a) $B_{Z}$ in the xy plane overlayed on fast gated fluorescence images. (b) $B_{Z}$ in the xz plane. (c) $J_{Y}$ in the xy plane. (d) $J_{Y}$ in the xz plane. 

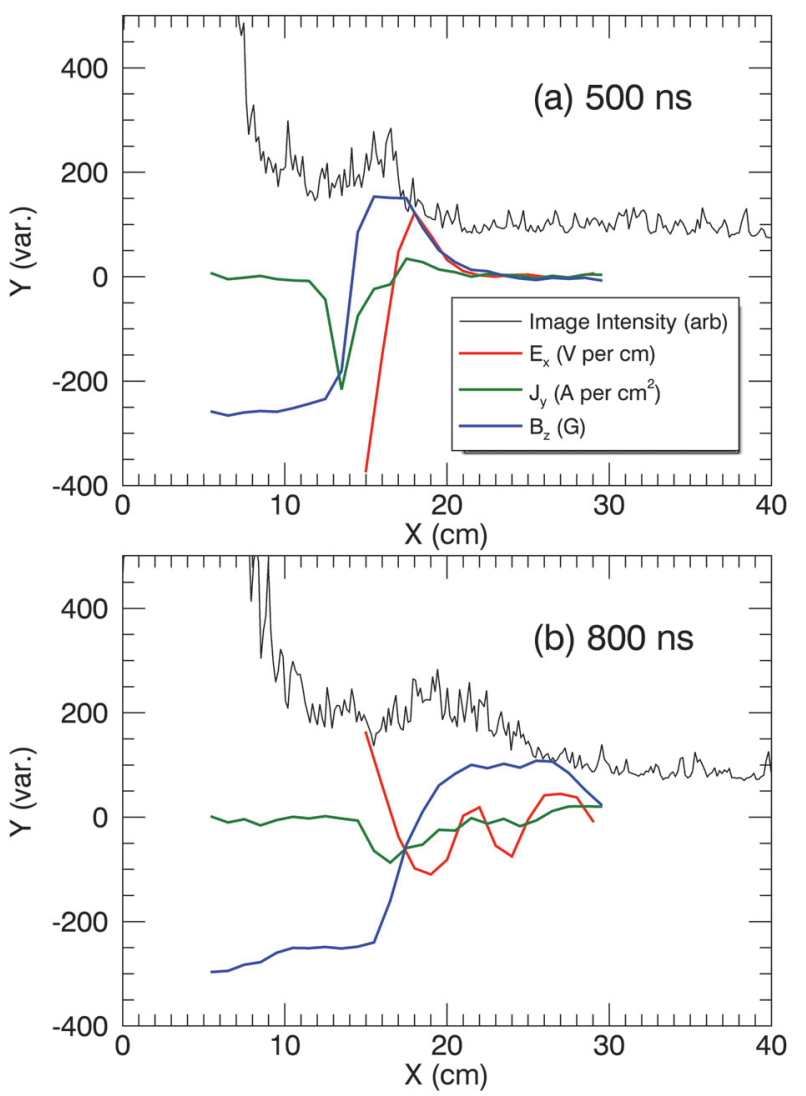

Figure 4: 1D comparisons of image intensity to field data at (a) $500 \mathrm{~ns}$ and (b) $800 \mathrm{~ns}$. The local intensity maximum near $20 \mathrm{~cm}$ corresponds to the shell in the 2D images. line, $N_{n}$ is the density of state $n$, and $d$ is the thickness of the plasma layer being integrated through. The state density $N_{n}$ is only a fraction of the total particle density. If this ratio were known (along with the transition probability and the plasma geometry), fluorescence intensity could be directly related to the particle density.

For a species of ions in local thermal equilibrium (LTE), the relative density of two states as a function of local ion temperature, $T_{i}$, can be calculated by use of the Boltzmann equation. For ions out of LTE (as likely present in this experiment), more detailed temperature or density information is necessary to determine the state distribution. Limited density and temperature information was collected in this experiment, restricting the scope of this analysis.

Time steps 200 ns, 500 ns, and 800 ns after the laser pulse in both planes are representative of different stages in the evolution of the debris-ambient interaction (Fig. 2a-b). The features of the images are similar in both the xy and xz planes. At early time (200 ns), intensity is concentrated in a bright region near the target, and is possibly continuum emission. At later time, a dim shell feature is seen propogating away from the main plume at $\approx 2.2 \times 10^{5} \mathrm{~m} / \mathrm{s}$. Comparison with field data (see Fig. 4) and filtered images (Fig 2c) suggest that this shell represents a population of carbon ions at the leading edge of the LPP. By 500 ns, the shell has fully separated from the bright region near the target. The shell expands faster than predicted by the Sedov-Taylor blastwave model [14], which is known to underestimate the expansion speed at low mach numbers [15], which is the case here (Alfvénic mach number $M_{A} \approx 1.6$ ).

Beginning around 300 ns the shell begins to decelerate by $3 \times 10^{12} \mathrm{~m} / \mathrm{s}^{2}$ (measured by time of flight), and instabilities grow along its edge $[16,17]$. In the xy plane, these instabilities are visible as fingers or flutes pointing radially outward. The flutes grow exponentially with a growth rate of $6 \times 10^{6} \mathrm{~s}^{-1}$ (measured frame-by-frame in the xy plane), consistant with the large Larmor radius limit of the Rayleigh-Taylor instability $[18,8]$. The flutes reach their maximum extent by $500 \mathrm{~ns}$, then are visible for a further several hundred nanoseconds before they dissipate by $800 \mathrm{~ns}$.

Filtered imaging at the CV $494 \mathrm{~nm}$ line (Fig. 2c) shows that the majority of $\mathrm{CV}$ fluorescence comes from the bright region near the target, although some does originate in the shell. As time progresses, 
the ions in the bright region move forward and eventually their fluorescence signal dissipates. The same process is observed in CIV and CIII, although delayed by $\approx 50 \mathrm{~ns}$ and $\approx 200 \mathrm{~ns}$ respectively. These images confirm that the bright shell in the unfiltered images is, in part, due to self-emission of $\mathrm{CV}$ ions.

The measured magnetic field, electric field, and current are dominated by the components $B_{z}, E_{x}$, and $J_{y}$. The diamagnetic cavity (the region in the xz plane where $B_{z}=0$ ) overlaps the area between the target and the shell. Comparison of data along the blowoff $(+\hat{x})$ axis (Fig. 4$)$ shows that the magnetic field pulse overlaps the intensity shell, while the local electric field maximum precedes the shell. A weak current sheet forms in front of the shell while a stronger current forms behind the shell. The observed separation between the current sheets and the fluorescence shell suggests that the observed increased fluorescence is caused by a higher density of carbon ions in that region, rather than excitation due to current. The overlap between the magnetic field maximum and the intensity shell suggests that this intensity feature may be used to track the magnetic field maximum in future experiments.

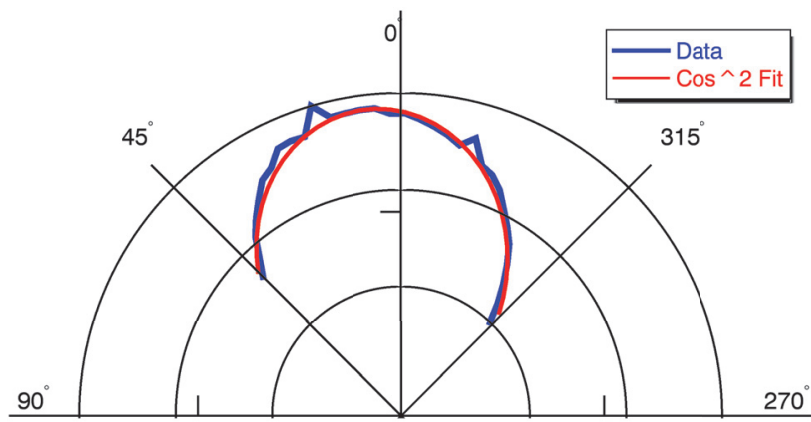

Figure 5: The initial velocity distribution in the $\mathrm{xz}$ plane, calculated using averaged images from 100-180 ns, follows a $\cos ^{2}$ distribution.

The relative fluorescence intensity in the images can also be used to infer the motion of the ablated plasma. The $2 \mathrm{D}$ nature of the images taken over several time steps allowed both the radial and angular velocity distributions to be calculated by time of flight. The initial angular velocity distribution of the LPP is of interest for comparison with simulation initializations [9]. The angular velocity distribution based on fluorescence images was measured in the perpendicular plane from 100-180 ns. The edge of the front was defined to be $4-5$ standard de- viations ahead of a Gaussian fit to the local intensity maximum corresponding to the shell feature. This calculation was repeated at different angles to find that the angular velocity distribution goes as $\cos ^{2}(\theta)$ (Fig. 5).

\section{Conclusion}

Fast gated fluorescence images of the interaction of a LPP and a background ambient plasma were recorded with and without optical filters, along with 2D planes of electric and magnetic field data. Fluorescence images showed the formation of a bright region near the target (coincident with a diamagnetic cavity) and a thin shell intensity feature propagating away from the target. The thin intensity shell is followed by an electric field maximum and coincident with a magnetic field maximum. Weak and strong current layers form ahead and behind the shell respectively. Finger instabilities were observed to grow as the shell decelerates with a growth rate consistent with the large Larmor radius limit of the Rayleigh-Taylor instability. Relative fluorescence intensity was also used to track the motion of the shell feature in time in order to calculate the initial angular velocity distribution. Fast gated fluorescence images analyzed by time of flight techniques and comparisons to large planes of field data provide insight into the role of particle distributions even without sufficient information to calculate the exact particle density. Future work will examine similar structures in quasi-parallel and parallel geometries.

\section{Acknowledgments}

This work was supported by the DTRA under Contract No. HDTRA1-12-1-0024, the DOE under Contract Nos. DE-SC0006538:0003 and the NSF Award No. 1414591. Experiments were performed at the UCLA Basic Plasma Science Facility (BaPSF), supported by the DOE and the NSF. We would like to thank the staff of BaPSF, Z. Lucky and M. Drandell, for their help conducting this experiment.

\section{References}

[1] D. S. Spicer, S. P. Maran, R. Clark, A model of the pre-Sedov expansion phase of supernova remnantambient plasma coupling and X-ray emission from SN 1987A, The Astrophysical Journal 356 (1990) 549-571. doi:10.1086/168862. 
[2] L. F. Burlaga, R. M. Skoug, C. W. Smith, D. F. Webb, T. H. Zurbuchen, A. Reinard, Fast ejecta during the ascending phase of solar cycle 23: Ace observations, 1998-1999, Journal of Geophysical Research: Space Physics 106 (A10) (2001) 20957-20977. doi:10.1029/2000JA000214.

[3] R. P. Drake, The design of laboratory experiments to produce collisionless shocks of cosmic relevance, Physics of Plasmas 7 (11) (2000) 4690-4698.

[4] C. Niemann, W. Gekelman, C. G. Constantin, E. T. Everson, D. B. Schaeffer, A. S. Bondarenko, S. E. Clark, D. Winske, S. Vincena, B. V. Compernolle, P. Pribyl, Observation of collisionless shocks in a large currentfree laboratory plasma, Geophysical Research Letters 41 (2014) 7413-7418.

[5] T. P. Wright, Earlytime model of laser plasma expansion, Physics of Fluids 14 (9) (1971) 1905-1910.

[6] D. B. Schaeffer, E. T. Everson, A. S. Bondarenko, S. E. Clark, C. G. Constantin, S. Vincena, B. V. Compernolle, S. K. P. Tripathi, W. Gekelman, C. Niemann, Laser-driven, magnetized quasi-perpendicular collisionless shocks on the large plasma device, Phys. Plasmas 21.

[7] A. Neogi, R. K. Thareja, Laser-produced carbon plasma expanding in vacuum, low pressure ambient gas and nonuniform magnetic field, Physics of Plasmas 6 (1).

[8] A. Collette, W. Gekelman, Structure of an exploding laser-produced plasma, Phys. Rev. Lett. 105 (2010) 195003. doi:10.1103/PhysRevLett.105.195003.

[9] S. E. Clark, E. T. Everson, D. B. Schaeffer, A. S. Bondarenko, C. G. Constantin, C. Niemann, D. Winske, Enhanced collisionless shock formation in a magnetized plasma containing a density gradient, Phys. Rev. E 90 (2014) 041101. doi:10.1103/PhysRevE.90.041101.

[10] W. Gekelman, P. Pribyl, Z. Lucky, M. Drandell, D. Leneman, J. Maggs, S. Vincena, B. Van Compernolle, S. K. P. Tripathi, G. Morales, T. A. Carter, Y. Wang, T. DeHaas, The upgraded large plasma device, a machine for studying frontier basic plasma physics, Review of Scientific Instruments 87 (2). doi:http://dx.doi.org/10.1063/1.4941079.

[11] E. T. Everson, P. Pribyl, C. G. Constantin, A. Zylstra, D. Schaeffer, N. L. Kugland, C. Niemann, Design, construction, and calibration of a three-axis, highfrequency magnetic probe b-dot probe... as a diagnostic for exploding plasmas, Rev. Sci. Insturm. 11.

[12] M. J. Martin, J. Bonde, W. Gekelman, P. Pribyl, A resistively heated ceb6 emissive probe, Review of Scientific Instruments 86 (5). doi:http://dx.doi.org/10.1063/1.4921838.

[13] F. E. Irons, N. J. Peacock, A spectroscopic study of the recombination of $c^{6+}$ to $c^{5+}$ in an expanding laserproduced plasma, Journal of Physics B: Atomic Molecular Physics 7 (15).

[14] G. Taylor, The formation of a blast wave by a very intense explosion. i. theoretical discussion, Proceedings of the Royal Society of London. Series A, Mathematical and Physical Sciences 201 (1065) (1950) 159-174.

[15] M. Hendijanifard, D. A. Willis, An improved method to experimentally determine temperature and pressure behind laser-induced shock waves at low mach numbers, Journal of Physics D: Applied Physics 44 (14) (2011) 145501.

[16] G. Dimonte, L. G. Wiley, Dynamics of exploding plasmas in a magnetic field, Physical Review Letters 67 (13)
(1991) 1755-1758.

[17] B. H. Ripin, J. D. Huba, E. A. McLean, C. K. Manka, T. Peyser, H. R. Burris, J. Grun, Sub-alfvenic plasma expansion, Physics of Fluids B 5 (10).

[18] J. D. Huba, J. G. Lyon, A. B. Hassam, Theory and simulation of the rayleigh-taylor instability in the limit of large larmor radius, Phys. Rev. Lett. 59 (1987) 29712974. doi:10.1103/PhysRevLett.59.2971. 